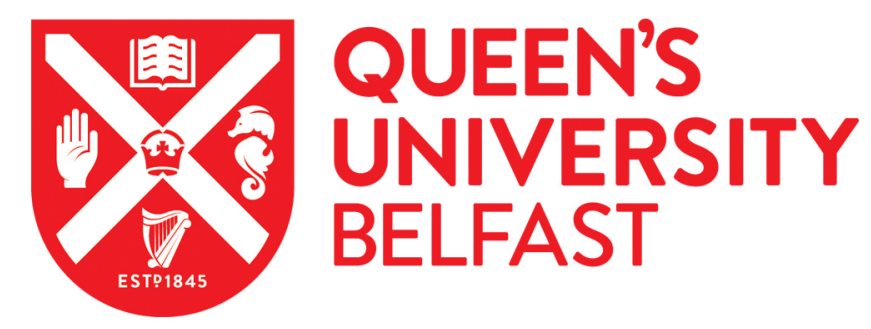

\title{
A Comparative Analysis of the Osteogenic Potential of Dental Mesenchymal Stem Cells
}

Winning, L., El Karim, I. A., \& Lundy, F. T. (2019). A Comparative Analysis of the Osteogenic Potential of Dental Mesenchymal Stem Cells. Stem Cells and Development, 28(15), 1050-1058.

https://doi.org/10.1089/scd.2019.0023

\section{Published in:}

Stem Cells and Development

\section{Document Version:}

Peer reviewed version

Queen's University Belfast - Research Portal:

Link to publication record in Queen's University Belfast Research Portal

\section{Publisher rights}

Copyright 2019 Mary Ann Liebert. This work is made available online in accordance with the publisher's policies. Please refer to any applicable terms of use of the publisher

\section{General rights}

Copyright for the publications made accessible via the Queen's University Belfast Research Portal is retained by the author(s) and / or other copyright owners and it is a condition of accessing these publications that users recognise and abide by the legal requirements associated with these rights.

Take down policy

The Research Portal is Queen's institutional repository that provides access to Queen's research output. Every effort has been made to ensure that content in the Research Portal does not infringe any person's rights, or applicable UK laws. If you discover content in the Research Portal that you believe breaches copyright or violates any law, please contact openaccess@qub.ac.uk. 


\section{A Comparative Analysis of the Osteogenic Potential of Dental Mesenchymal Stem Cells.}

Lewis Winning ${ }^{1}$, Ikhlas A. El Karim ${ }^{1}$, Fionnuala T. Lundy ${ }^{1 *}$.

${ }^{1}$ The Wellcome-Wolfson Institute for Experimental Medicine, School of Medicine, Dentistry and Biomedical Sciences, Queen's University Belfast, Northern Ireland.

Key words: Periodontal ligament stem cells; Osteoblastic differentiation; Alkaline phosphatase; Alizarin red staining; qPCR.

Running title: Osteogenic potential of PDLSC

* - Corresponding author:

Dr. Fionnuala T. Lundy

The Wellcome-Wolfson Institute for Experimental Medicine

School of Medicine, Dentistry and Biomedical Sciences

Queen's University Belfast

97 Lisburn Road

Belfast

BT9 7BL

Northern Ireland

Email: f.lundy@qub.ac.uk

Phone: +44 (0)28 90976387

\section{Author Disclosure Statement}

The authors declare that there are no conflicts of interest. The study was supported by a research grant from the British Society of Periodontology. 


\section{Author details:}

\section{Dr Lewis Winning}

The Wellcome-Wolfson Institute for Experimental Medicine

School of Medicine, Dentistry and Biomedical Sciences

Queen's University Belfast

97 Lisburn Road

Belfast

BT9 7BL

Northern Ireland

Email: 1.winning@qub.ac.uk

Phone: $+44(0) 2890976387$

\section{Dr Ikhlas El Karim}

The Wellcome-Wolfson Institute for Experimental Medicine School of Medicine, Dentistry and Biomedical Sciences

Queen's University Belfast

97 Lisburn Road

Belfast

BT9 7BL

Northern Ireland

Email: $\underline{\text { i.elkarim@qub.ac.uk }}$

Phone: +44 (0)289097 6026

\section{Dr. Fionnuala T. Lundy}

The Wellcome-Wolfson Institute for Experimental Medicine

School of Medicine, Dentistry and Biomedical Sciences

Queen's University Belfast

97 Lisburn Road

Belfast

BT9 7BL

Northern Ireland

Email: f.lundy@qub.ac.uk

Phone: +44 (0)289097 6026

\section{Authors all contributed equally to this work.}

\section{Prior conference presentation:}

This paper was presented at the 96th General Session of the International Association for Dental Research conference, London, UK, July $28^{\text {th }} 2018$. 


\section{Abstract}

The aim of this study was to compare the in-vitro osteogenic differentiation potential of withinsubject mesenchymal stem cells (MSCs) derived from the dental pulp of permanent teeth (dental pulp stem cells - DPSCs), the dental pulp of deciduous teeth (pulp of human exfoliated deciduous teeth - SHEDs), and the periodontal ligament of permanent teeth (periodontal ligament stem cells - PDLSCs). A single subject was identified that required concurrent removal of both deciduous and permanent teeth for orthodontic purposes. Primary, mixed population cells from dental pulp, deciduous dental pulp, and periodontal ligament were obtained by the tissue out-growth method. Subsequently, isolation of STRO-1 +ve cells from their respective primary cell cultures was achieved by immunomagnetic separation. Cells were induced with an osteogenic cocktail of $5 \mathrm{mM} \beta$-glycerophosphate, $100 \mathrm{nM}$ dexamethasone and $50 \mathrm{mg} / \mathrm{mL}$ ascorbic acid for up to 21 days. Osteogenic responses were assessed functionally by an alkaline phosphatase (ALP) activity assay and an alizarin red staining assay. Expression of the early osteogenic associated genes alkaline phosphatase gene (ALPL), runt-related transcription factor 2 (RUNX2), collagen type I alpha 1 (COL1A), and secreted phosphoprotein 1 (SPP1) were compared by qPCR at days 1, 4 and 7 of differentiation. Functional analysis revealed there were significant differences in intracellular ALP activity on days $4,7,10 \& 14$ with PDLSCs $>$ SHEDs $>$ DPSCs. Quantification of alizarin red staining showed significantly more mineralisation for PDLSCs by day 21. Gene expression analysis showed significant early up-regulations of the osteogenic markers ALPL and COL1A1 for PDLSCs over DPSCs and SHEDs. SHEDs showed significantly higher upregulation of ALPL over DPSCs. In conclusion, PDLSCs showed a significantly higher osteogenic differentiation potential than both DPSCs and SHEDs evidenced by functional studies and gene expression. This may be of significance for the use of dentally derived MSCs in bone tissue engineering applications. 


\section{Introduction}

Mesenchymal stem cells (MSCs) are recognised as offering much promise in the areas of tissue engineering and regenerative medicine due to their ability to differentiate into specific tissue forming cell types. The current practice of tissue engineering involves the combination of cells and/or proteins with biomaterials to generate a new tissue [1]. Factors affecting the success of tissue engineering include: the type of stem cell utilised; the strategies used to isolate and expand the specific cells; and the choice of the biomaterial used as a scaffold [2]. MSCs have great potential in bone-tissue engineering applications due to their ability to undergo osteoblastic differentiation under the appropriate conditions. Bone marrow-derived mesenchymal stem cells (BMMSCs) are currently considered the gold standard MSC for bone tissue engineering [3]. Intense research effort has been directed towards examining BMMSCs for applications in bone-tissue engineering $[4,5]$.

As well as bone marrow, MSC populations have also been isolated from several oral tissues such as dental pulp, periodontal ligament, apical papilla, dental follicle, tooth germ, gingivae, and periosteum [6-10]. Owing to their relative ease of access and less invasive harvesting techniques in comparison to BMMSCs, dental MSCs represent an attractive alternative MSC source for bone-tissue engineering applications [11, 12]. Studies have also found other advantageous properties such as the ability to produce higher colony forming units, a higher cell proliferation rate, and a longer survival time in comparison to BMMSCs [13, 14]. A number of studies have demonstrated the osteogenic potential of the various dental MSCs utilising both in-vitro and in-vivo models [15]. Whilst an osteogenic potential in general may have been demonstrated, it should be realised there are significant phenotypic differences between the different types of dental MSCs reflecting their distinct functional properties [16, 
17]. This heterogeneity may potentially alter the efficacy of the individual lineage's osteogenic potential. To this end, several studies have been performed attempting to compare the osteogenic potential of various selected dental MSCs.

A study investigated the osteogenic potential of periodontal ligament stem cells (PDLSCs) compared to that of stem cells from the pulp of human exfoliated deciduous teeth (SHEDs) [18]. Results showed that both cell types were capable of undergoing osteogenic induction, however, there was a significantly higher cell proliferation of PDLSC over SHEDs, which resulted in greater calcium deposition after 3-weeks of culture. The authors suggested PDLSCs were a superior stem cell source for osteogenic purposes. Interpretation may be limited due to the use of cells from different donors, results may therefore reflect inter-individual variations. The osteogenic differentiation potential of human dental pulp stem cells (DPSCs) has previously been compared with the osteogenic differentiation potential of stem cells from the apical papilla (SCAPs) [19]. Both DPSC and SCAP cell populations derived from impacted third molars within the same subject. Results demonstrated that although both DPSCs and SCAP were able to differentiate into osteo/odontoblast-like cells, SCAPs had a significantly higher mineralisation potential. In a study comparing DPSCs with SHEDs [20], cells were collected and harvested from different donors. SHEDs displayed a higher osteogenic potential over DPSCs evidenced by significantly higher calcium deposition, and a significantly higher expression of the osteogenic related genes ALPL (alkaline phosphatase gene), RUNX2 (runtrelated transcription factor 2), and COL1A1 (collagen, type I, alpha 1). The in-vivo comparisons showed that SHED transplants produced bone-like structures, whereas DPSC transplants formed connective tissue with comparatively smaller amounts of mineralized tissue. A studying comparing the osteogenic differentiation potential between DPSCs and PDLSCs has previously been carried out [21]. To eliminate inter-subject differences, they harvested and isolated DPSCs and PDLSCs from the same tooth for comparison (one subject). 
Results overall were inconclusive; PDLSCs had significantly higher COL1A1 but lower OCN (Osteocalcin) gene expression. Mineralisation staining assays suggested greater mineralisation for DPSC cultures, although no quantification was carried out.

In summary, there is a lack of evidence to recommend a specific dental MSC lineage for bonetissue engineering purposes. A thorough one-to-one comparison of dental MSCs from a single donor for key osteogenic properties has not been evaluated in detail. It is important to understand whether MSCs derived from close tissues behave differently and have a different osteogenic potential. Possible differences may have implications for dental MSC bone tissue engineering applications. The aim of this study therefore, was to compare the in-vitro osteogenic differentiation potential of mesenchymal stem cells derived from the dental pulp of a permanent tooth (DPSCs), the dental pulp of deciduous teeth (SHEDs), and the periodontal ligament of a permanent tooth (PDLSCs). 


\section{Materials and Methods}

\section{Study population}

Ethical approval was granted by the Office for Research Ethics Northern Ireland, for the collection of teeth from the School of Dentistry, Belfast Health and Social Care Trust (Ethical approval number 08/NIR03/15). Following written consent, subjects were identified that required concurrent removal of both deciduous and permanent teeth for orthodontic purposes from a similar region of the oral cavity. Teeth were caries and periodontal disease free. To rule out potential differences which may be attributable to within-subject variation, experiments were based on explants from a single donor where dental pulp from a deciduous tooth, dental pulp from a permanent tooth, and periodontal ligament from a permanent tooth were all successfully explanted and expanded. In this case, a systemically healthy, 16-year-old Caucasian male, who required orthodontic extraction of an upper left first permanent premolar and the adjacent upper left second deciduous molar teeth due to orthodontic crowding.

\section{Explant, culture, and isolation of dental MSC lineages}

Primary, mixed population cells from dental pulp, deciduous dental pulp, and periodontal ligament were obtained by the tissue out-growth method. Briefly, extracted teeth were rinsed with sterile phosphate-buffered saline (PBS, $0.01 \mathrm{M}, \mathrm{pH}$ 7.4, Invitrogen, UK). The periodontal ligament (PDL) tissue from the middle one third of the permanent premolar root was then scraped off with a sterile scalpel [22]. This PDL tissue was then cut into pieces (approximately $1 \mathrm{~mm} \times 1 \mathrm{~mm} \times 1 \mathrm{~mm}$ ) and placed into a $25 \mathrm{~cm}^{2}$ culture flask (Falcon). For pulp tissue (permanent and deciduous teeth), the tooth was split open with a bench fitted vice with a cutting edge [23]. The pulp tissue was then carefully removed with a size $40 \mathrm{~K}$ Flexofile (Dentsply, 
Maillefer, Switzerland). This pulp tissue was then similarly minced into tiny pieces (approximately $1 \mathrm{~mm} \times 1 \mathrm{~mm} \times 1 \mathrm{~mm}$ ) and placed into a T25 culture flask. Cell explants were grown and expanded under identical culture conditions. The culture media consisted of alphamem supplemented with 10\% fetal bovine serum, 1\% penicillin/streptomycin (all Invitrogen, UK), 1\% L-glutamine, and 20mM ascorbic acid (both Sigma-Aldrich, UK). Samples were cultured in an incubator at $37^{\circ} \mathrm{C}$ in $5 \% \mathrm{CO}_{2}$, with the media being changed every 3 days until the primary cells migrated from the tissue explant remnants and reached confluence. The primary mixed cell populations were subsequently passaged, prior to harvesting a subpopulation of stem cells.

Isolation of STRO-1 +ve stem cells from their respective primary cell cultures was achieved by immunomagnetic separation [12]. Briefly, magnetic bead-linked rat anti-mouse IgM (Dynal, Oslo, Norway) beads were incubated with mouse IgM anti-human STRO-1 (Invitorgen, UK). These were then incubated with the different cell types in an isolation buffer for 20 minutes at $4^{\circ} \mathrm{C}$. A magnetic cell sorting rack (DynaMag, Invitrogen, UK) was used to discard cells not magnetically retained. Thus three, STRO-1 +ve, stem cells populations were derived (DPSCs, SHEDs, and PDLSCSs).

Cells were cultured in a 'control media' consisting of $10 \%$ Fetal bovine serum, $1 \%$ penicillin/streptomycin, 1\% L-Glutamine, and $50 \mathrm{mg} / \mathrm{mL}$ ascorbic acid. In experiments requiring osteogenic induction an 'osteogenic media' with the additional additives of $5 \mathrm{mM} \beta$ glycerophosphate and 100nM dexamethasone was utilised. Media was changed every other day. Cells from 'passage 4' were used in all experiments. 


\section{Alkaline phosphatase activity}

Each of the three cell types (DPSC, SHED, \& PDLSCs) were seeded in triplicate in 12-well plates at a density of $4 \times 10^{4}$ cells $/ \mathrm{mL}$ and grown to $\sim 70 \%$ confluence. Cells were then induced with the osteogenic media (time zero). Osteogenic responses were assessed functionally by an intra-cellular alkaline phosphatase (ALP) activity assay on days $1,4,7,10$, and 14 . At the designated time point, cells were rinsed with PBS twice, and then lysed by adding $150 \mu$ l of lysis buffer $(0.2 \%$ Triton $\mathrm{X}-100)$. This was followed by a 15 minute incubation period at $37^{\circ} \mathrm{C}$. Wells were then scraped and the lysate transferred to $1.5 \mathrm{ml}$ tubes, before being vortexed and centrifuged at $10,000 \mathrm{rpm}$ at $4^{\circ} \mathrm{C}$ for 10 minutes. The supernatant of each sample was collected for ALP quantification using a diethanolamine detection kit (Sigma-Aldrich, UK) utilising pnitrophenyl phosphate (PNPP) as the substrate. Values were normalised to total protein content, which was measured using a bicinchoninic acid (BCA) protein assay kit (Pierce, Rockford, IL, USA) and expressed as $\eta \mathrm{mol} \mathrm{PNPP} / \mathrm{min} / \mathrm{mg}$ protein.

\section{Alizarin Red Staining}

Each of the three cell types were seeded in triplicate in 6-well plates at a density of $4 \times 10^{4}$ cells $/ \mathrm{mL}$ and grown to $\sim 70 \%$ confluence. Alizarin red (AR) staining was carried out at days 7 , 14 and 21. For each time point triplicate wells were designated as either 'Control media' or 'Ostegogeic media'. At the corresponding time point, cells were rinsed with PBS (Dulbecco's phosphate buffered saline, without $\mathrm{Ca}^{2+} / \mathrm{Mg}^{2+}$, PromoCell, UK) twice and subsequently fixed in $70 \%$ ice cold ethanol for $30 \mathrm{~min}$. After three washes with distilled water $\left(\mathrm{diH}_{2} \mathrm{O}\right)$, the cells were stained for 30 min with 2\% alizarin red, $\mathrm{pH} 4.2$ (Sigma-Aldrich, UK). Cells were then washed five times with $\mathrm{diH}_{2} \mathrm{O}$. Full well images and images at x10 magnification were captured with an inverted microscope with integrated camera (Leica, UK). 
Quantification of the total mineralized tissue produced per well was performed by extracting the AR from the stained sites by adding $2 \mathrm{ml}$ of cetylpyridinium chloride (CPC, Sigma-Aldrich, UK) buffer $\left(10 \%\right.$, w/v) in $10 \mathrm{mM} \mathrm{Na} \mathrm{HPO}_{4}(\mathrm{pH} 7)$ for $1 \mathrm{~h}$ at $37^{\circ} \mathrm{C}$. Subsequently, $100 \mathrm{ml}$ aliquots were transferred to a 96 -well plate and the $\mathrm{OD}_{570 \mathrm{~nm}}$ of the solution was measured using a microplate reader (Tecan GENios, MTX Lab systems, US). Values were expressed as fold change in optical density $\left(\mathrm{OD}_{570 \mathrm{~nm}}\right)$ of osteogenic wells over control wells for each cell type at that time point.

\section{Osteogenic gene expression}

Each of the three cell types were seeded in triplicate in 12 well plates at a density of $4 \times 10^{4} / \mathrm{ml}$ and grown to $70 \%$ confluence. Cells were then induced with the osteogenic media (time zero). At days 1, 4 and 7 RNA extraction and cDNA synthesis were carried out. Briefly, wells were rinsed twice with cold phosphate-buffered saline and RNA extracted using the RNeasy mini kit (Qiagen, UK) according to the manufacturer's instructions. RNA yield and purity was assessed on a Take3 plate (BioTek, USA). Following this, cDNA was synthesised using a SuperScript vilo cDNA synthesis kit (Invitrogen, UK) and frozen $\left(-80^{\circ} \mathrm{C}\right)$. Real-time qPCR for alkaline phosphatase gene (ALPL), collagen type I alpha I gene (COL1A1), runt-related transcription factor gene (RUNX2), and secreted phosphoprotein 1 gene (SPP1) was carried out on a Stratagene PCR machine (Agilent Technologies). Normalisation of results was achieved by using the house-keeping genes Beta-2 microglobulin (B2M) and glucuronidase beta (GUSB). Primer sequences are listed in Table 1. Sequence amplification was performed under the following conditions: denaturation at $55^{\circ} \mathrm{C}$ for 2 minutes and $95^{\circ} \mathrm{C}$ for 10 minutes. This was then followed by 45 cycles of $95^{\circ} \mathrm{C}$ for 30 seconds and $50^{\circ} \mathrm{C}$ for 1 minute. The relative gene expression was calculated by the $2^{-\Delta \Delta \mathrm{CT}}$ method. Results are reported as fold change in gene expression relative to the day 1 DPSC. 


\section{Statistical analysis}

ALP activity data and qPCR data with multiple groups at different time points, was analysed by a two-way Analysis of Variance (ANOVA) test followed by Tukey's post hoc test for multiple comparisons. The AR staining assay was analysed using one-way ANOVA followed by Tukey's post hoc test for multiple comparison for each separate time point. Experiments were performed in triplicate and repeated three times. The level of statistical significance was set at $p<0.05$. The statistical analyses were performed with IBM SPSS Statistics for Windows (IBM Corp. Released 2012. Version 21.0. Armonk, NY). Graphs were constructed using Prism version 7.00 for Windows (GraphPad Software, La Jolla California USA, www.graphpad.com). 


\section{Results}

Three different dental soft tissues (dental pulp from a permanent tooth, dental pulp from a deciduous tooth, and periodontal ligament from a permanent tooth) from a single donor were successfully explanted by the tissue out growth method. Isolation of STRO-1 +ve cells by immunomagnetic separation was then concurrently undertaken on cultures, to produce three distinct populations: DPSCs; SHEDs; and PDLSCs.

To screen for the osteogenic potential of the 3 cell groups, ALP activity was measured at 1, 4, 7 and 14 days (Figure 1). At day 1 there was minimal ALP activity in all three cultures with no significant differences. By Day 4, there was a significant increase in ALP activity for PDLSCs, compared to both DPSCs and SHEDs. By day 7, PDLSCs continued to have significantly greater ALP activity than both types of pulp stem cells, however SHED ALP activity was now significantly greater than DPSC. As time progressed ALP activity for all three cell types increased. A similar pattern of significantly greater ALP activity for PDLSC $>$ SHED $>$ DPSC continued at both days 10 and 14.)

AR staining was used to ascertain the presence of mineralised nodules formed by the 3 cell osteogenic lineages (Figures 2 \& 3). At day 7 there were subtle differences between the cell lineages cultured in a control media versus those in an osteogenic media. Those in the osteogenic media showed enhanced proliferation with a greater uptake of back ground AR staining. No nodular formation was observed at this time point. There were no significant differences in quantification of staining between each of the cell type, (Figure 4A). By day 14 there was noticeable nodule formation in the PDLSC osteogenic culture. Quantification 
showed significantly greater staining in PDLSC $>$ SHED $>$ DPSCs, (Figure 4B). By day 21, there was gross mineralisation of the whole well for the PDLSC osteogenic culture. Nodule formation was now visible in the SHED osteogenic culture. Evidence of very early nodule formation was observed at magnification for the DPSC osteogenic culture. Also of note, was the early nodule formation at magnification in the control PDLSC culture. Quantification again showed significantly greater staining in PDLSC > SHED > DPSCs, (Figure 4C).

The osteogenic phenotype of DPSCs, SHEDs and PDLSCs following culture in an osteogenic media was confirmed at the transcriptional level by genes encoding ALP, COL1A1, RUNX2, and SPP1, (Figure 4). Across the 4 genes studied the ALPL gene expression provided the most interpretable information to what was being observed functionally: There were no significant differences in gene expression at Day 1. At day 4 there was a stepped pattern with PDLSCs having a x8.6 fold increase in ALPL gene expression, SHEDs a x4.1 increase, and DPSC x1.3. By day 21 there was a x25.2 fold increase for PDLSCs, x8.5 fold increase for SHEDs, and a x3.0 fold increase for DPSCs. Also of note, was the gene expression for COL1A1. PDLSC showed a modest early upregulation of COL1A1 with a x2.7 fold increase versus $\sim$ x1.0 for both types of pulp stem cell. A similar pattern was observed at Day 4 with significant greater upregulation of x3.7 for PDLSCs, although expression in DPSCs and SHEDs had now increased to x1.8 for both. By day 7 upregulation for PDLSCs had fallen to x2.7, with no significant difference to DPSCs (x2.5). Both DPSCs and PDLSCs had significantly greater expression than SHEDs (x1.8). Although statistically significant differences were observed for both RUNX2 and SPP1, fold change values were small $(<2.0)$ across groups. 


\section{Discussion}

The results of this study suggest that the in-vitro osteogenic potential of PDLSCs is superior to SHEDs which is in turn superior to DPSCs, (PDLSCs $>$ SHEDs $>$ DPSCs). This was demonstrated at a functional level by intra cellular ALP enzyme activity and additionally by mineralised nodule deposition with AR staining. At a gene level, results reflecting the functional findings were observed for the osteogenic associated genes ALPL and COL1A1. This study provides evidence that despite the anatomical proximity of their tissues of origin, dental MSCs represent distinct MSC populations with respect to their in-vitro osteogenic potential. This may have implications for in-vivo dental MSC bone tissue engineering applications.

Although this is a novel study, comparability to results from previous studies can be inferred: Chadipiralla and co-workers showed that PDLSCs had a greater osteogenic potential than SHEDs [18]; and Wang and colleagues showed that SHEDs had a greater osteogenic potential than DPSCs [20]. The novelty of the present study relates to the fact all three cell populations were examined concurrently and a robust experimental design was employed. An important aspect of this design, was the use of a single donor to yield the three cell populations. Although it could be argued that the generalisability of the findings from a single study may be limited, this is somewhat outweighed by the numerous advantages offered by a single donor approach. In studies comparing efficacies of different cell types where cells have been harvested from different donors, it is difficult to determine if observed functional differences are indeed due to different cell phenotypes, or alternatively due to genetic variability relating to the use of different donors. Connected with this, if using donors of different ages, studies have shown that there is a donor age-related decrease in osteogenic differentiation capacity [24, 25]. Other 
factors which may potentially affect the osteogenic differentiation capacity when using multiple donors relate to: state of confluence; method of isolation; storage time and conditions; properties of culture media used; and incubation environments [19]. It has also been found that epigenetic and genetic alterations occur during culture expansion (including passaging), which can unequivocally affect osteoblastic lineage commitment/differentiation [26].

A clear pattern unfolded with regards to mineralisation and nodule formation by AR staining. PDLSCs readily formed nodules at day 14, and by day 21 there was complete mineralisation of the well. SHEDs, behind PDLSCs, had clear nodule formation by day 21, whereas DPSCs had only very early evidence of nodule formation when observed under x10 microscope. Of interest, PDLSCs kept in a control media also started to show signs of nodule formation at day 21. This is potentially triggered by the high adhesion forces developed amongst cells after prolonged confluency that make the monolayer contractile, behaving more like a tissue and conferring PDLSC's role to tissue homeostasis and repair. Quantification of the AR staining, showed a clear pattern with significantly greater staining uptake in PDLSC $>$ SHED $>$ DPSC osteogenic cultures at both days 14 and day 21.

Undifferentiated MSCs show weak ALP activity, whereas fully differentiated osteoblasts have higher activity [27]. The results indicate that PDLSCs had significantly higher ALP activity than both types of pulp stem cells at days 4, 7, 10 and 14. SHEDs had significantly greater ALP activity than DPSCs at days $7,10, \& 14$. The overall pattern observed suggests all three phenotypes were committing to an osteoblastic type lineage albeit at different rates: PDLSCs at a greater rate than SHEDs and DPSCs, and SHEDs at a greater rate than DPSCs. At the gene level, the ALPL gene codes for the production of alkaline phosphatase. The upregulation of 
alkaline phosphatase is generally regarded as an early marker of osteogenic differentiation. The results mirrored what was being observed at a functional level specifically for ALPL. At day 4 there was a stepped pattern with PDLSCs having a $\mathrm{x} 9$ fold increase in ALPL gene expression, SHEDs a $\mathrm{x} 4$ increase, and DPSC remaining at $\mathrm{x} 1$. By day 21 there was a $\mathrm{x} 25$ fold increase for PDLSCs, $\mathrm{x} 8$ fold increase for SHEDs, and a x3 fold increase for DPSCs. Also of note, was the gene expression for COL1A1 which codes for production of type I collagen and plays an important role in cell adhesion, proliferation and differentiation of the osteoblast phenotype. PDLSC showed a modest early upregulation of COL1A1 with a $\mathrm{x} 3$ fold increase versus $\mathrm{x} 1$ for both types of pulp stem cell. The interpretation of the gene expression for RUNX2 and SPP1 genes was inconclusive. Results of the osteogenic gene expression analysis add further evidence to what was being observed functionally: PDLSCs were further along the osteogenic differentiation pathway than SHEDs which were in turn further along than DPSCs.

A potential explanatory factor for the observed results relates to the likely heterogeneity of cells within a STRO-1 +ve sorted cell population. Heterogeneity within a sorted population may give rise to differences in proliferative capacity as well as multipotency [28]. No ideal technique of cell sorting has been identified that will result in perfectly homogenous populations of MSCs [29]. This is a recognised limitation across efficacy type studies comparing populations of MSCs derived from different tissues. STRO-1 +ve cells derived from the periodontal ligament in particular, may contain a subpopulation of STRO-1+ve preosteoblasts type cells [30], which could potentially contribute to increased ALP activity. However, given that starting values for ALP activity were comparatively low across all three cell types (Figure 1), this was unlikely to have been a major factor. 
Dental MSCs are known to be of ectomesenchymal origin and are considered to share a common lineage of being derived from neural crest cells [31]. Most dental MSCs identified thus far have generic MSC-like properties, including expression of marker genes and differentiation into mesenchymal cell lineages (osteoblasts, adipocytes and chondrocytes) invitro and, to some extent in-vivo [32]. Despite similarities across dental MSCs, phenotypic differences do exist. The difference in osteogenic potential found in the present study most likely relates to the phenotypic differences across PDLSCs, SHEDs, and DPSCs [17]. If we consider the tissue of origin of each of these types of cells, it would seem logical that PDLSCs should have a greater osteogenic potential due to the periodontal ligament's intimate relationship with alveolar bone. This would also agree with the premise that the function of PDLSCs is thought to be repair of damaged tissue including regeneration of lost alveolar bone [33]. Based on their anatomic location, it should follow that DPSCs would be more likely to follow an odontoblastic route (for dentine repair), rather than osteogenic route. Although several reports in the literature indicate the osteogenic potential of DPSCs [15], comparative gene expression studies with osteoblast-like cells generated from DPSCs and normal osteoblasts derived from BMMSCs indicated many distinct molecular differences [34]. A sensitivity analysis was performed to investigate gene expression of dentin sialophosphoprotein (DSPP) across the three cell types during osteogenic stimulation (Supplementary information S1). The DSPP gene provides instructions for synthesis of the protein dentin sialophosphoprotein, which is an important component of dentine. Relative to PDLSCS, the fold change expression of DSPP by both SHEDs and DPSCs was higher at Day 1 (x1.0 for PDLSCs, and x3.0 for both SHEDs and DPSCs), and at Day 7 (x2.2 for PDLSC, x5.8 for SHED, x4.4 for DPSC). This gives some indication that both DPSCs and SHEDs may have been committing to more of an odontoblastic or odnto/osteoblastic lineage compared to that of a predominantly osteoblastic lineage in PDLSCs. 
Mineralisation and osteogenesis from DPSCs also differ markedly from what has been reported with BMMSCs, with osteo-induced DPSCs resembling osteo-dentin rather than a typical osteoblast [17]. Tatullo and colleagues recently carried out a study comparing the osteogenic potential of DPSCs versus Periapical Cyst Mesenchymal Stem Cells (PCy-MSCs) [35]. Following osteogenic induction DPSCs seem to be directed towards dentinogenesis, whilst PCy-MSCs were directed towards osteogenesis.

SHEDs, considered immature DPSCs, do however have a robust osteogenic capacity and give rise to bone upon in-vivo transplantation, unlike DPSCs which give rise to dentin [9]. This may relate to a greater degree of 'stemness' and pluripotency in comparison to DPSCs [20]. Further to this, an important comparison between SHEDs and DPSCs is the significantly higher proliferation rate of SHEDs [36]. There is evidence to suggest that the expression of osteoblast related genes may occur faster in highly proliferative mesenchymal stem cell populations [37]. At a molecular level, differences in populations of MSCs derived from the pulp of both deciduous and permanent teeth have also been investigated [38]. Using specifically designed microarray panels, RT q-PCR, Western blot and immunohistochemistry it was found that HMGA2, a stem cell-associated gene, is robustly expressed in deciduous pulp cells but not in permanent tooth pulp cells. Additionally, several genes associated with mitosis were highly expressed in deciduous pulp cells, while matrix genes and several signalling molecules were more strongly expressed in the adult pulp cells. The authors concluded that based on molecular level findings. MSCs derived from deciduous teeth may be better suited for regenerative medicine and tissue engineering purposes than cells from permanent teeth [38].

A question raised based by this evidence, is, whether PDLSCs from deciduous teeth possess a greater osteogenic potential than those from permanent teeth. Khoshhal and colleagues, carried out a study comparing the osteogenic properties of PDLSCs from deciduous teeth versus PDLSCs from permanent teeth [39]. Teeth were sourced from different subjects, with 
osteogenic potential was assessed by AR staining and ALP activity. Results showed that following osteogenic induction, both isolated cell populations were able to form colonies and differentiate into osteoblasts. When comparing the two cell populations, there was a higher ALP activity in PDLSC cultures from permanent teeth. However, AR quantitative analysis revealed no statistically significant differences in mineralisation potential between the two cell types. The results of this study contrast with a study carried out by Silvério and colleagues [40]. Isolates of permanent PDLSCs and deciduous PDLSCs collected from different donors, were again compared for their osteogenic potential. Examining osteogenic gene expression by qPCR, they found higher mRNA levels for ALP and bone sialoprotein (BSP) expression in deciduous PDLSCs compared to permanent. This suggested a greater osteogenic potential of deciduous PDLSCs. The limited number of studies and ambiguity in results means no conclusion can be made on the osteogenic potential of PDLSCs from permanent teeth versus deciduous.

\section{Conclusion}

PDLSCs showed a significantly higher in-vitro osteogenic differentiation potential than both SHEDs and DPSCs evidenced by functional studies and gene expression. This may be of significance for the use of dental MSCs in bone tissue engineering applications. Further studies are warranted to validate whether these in-vitro results are replicated in an in-vivo environment. 


\section{References}

1. Leyendecker Junior A, CC Gomes Pinheiro, T Lazzaretti Fernandes, and D Franco Bueno. (2018). The use of human dental pulp stem cells for in vivo bone tissue engineering: A systematic review. Journal of tissue engineering. 9: $2041731417752766-2041731417752766$. 2. Langer R and JP Vacanti. (1993). Tissue engineering. Science. 260: 920-926.

3. Amini AR, CT Laurencin, and SP Nukavarapu. (2012). Bone tissue engineering: recent advances and challenges. Critical reviews in biomedical engineering. 40: 363-408.

4. Olivares-Navarrete R, SL Hyzy, JH Park, GR Dunn, DA Haithcock, CE Wasilewski, BD Boyan, and Z Schwartz. (2011). Mediation of osteogenic differentiation of human mesenchymal stem cells on titanium surfaces by a Wnt-integrin feedback loop. Biomaterials. 32: 6399-6411.

5. Rausch-Fan X, Z Qu, M Wieland, M Matejka, and A Schedle. (2008). Differentiation and cytokine synthesis of human alveolar osteoblasts compared to osteoblast-like cells (MG63) in response to titanium surfaces. Dent Mater. 24: 102-110.

6. Egusa H, W Sonoyama, M Nishimura, I Atsuta, and K Akiyama. (2012). Stem cells in dentistry--part I: stem cell sources. J Prosthodont Res. 56: 151-165.

7. Gronthos S, M Mankani, J Brahim, PG Robey, and S Shi. (2000). Postnatal human dental pulp stem cells (DPSCs) in vitro and in vivo. Proc Natl Acad Sci U S A. 97: 13625-13630. 8. Seo BM, M Miura, S Gronthos, PM Bartold, S Batouli, J Brahim, M Young, PG Robey, CY Wang, and S Shi. (2004). Investigation of multipotent postnatal stem cells from human periodontal ligament. Lancet. 364: 149-155.

9. Miura M, S Gronthos, M Zhao, B Lu, LW Fisher, PG Robey, and S Shi. (2003). SHED: stem cells from human exfoliated deciduous teeth. Proc Natl Acad Sci U S A. 100: 58075812 .

10. Sonoyama W, Y Liu, D Fang, T Yamaza, BM Seo, C Zhang, H Liu, S Gronthos, CY Wang, S Wang, and S Shi. (2006). Mesenchymal stem cell-mediated functional tooth regeneration in swine. PLoS One. 1: e79.

11. Morsczeck C, G Schmalz, TE Reichert, F Vollner, K Galler, and O Driemel. (2008). Somatic stem cells for regenerative dentistry. Clin Oral Investig. 12: 113-118.

12. Winning L, L Robinson, AR Boyd, IA El Karim, FT Lundy, and BJ Meenan. (2017). Osteoblastic differentiation of periodontal ligament stem cells on non-stoichiometric calcium phosphate and titanium surfaces. J Biomed Mater Res A. 105: 1692-1702.

13. Morad G, L Kheiri, and A Khojasteh. (2013). Dental pulp stem cells for in vivo bone regeneration: a systematic review of literature. Arch Oral Biol. 58: 1818-1827.

14. Bright R, K Hynes, S Gronthos, and PM Bartold. (2015). Periodontal ligament-derived cells for periodontal regeneration in animal models: a systematic review. J Periodontal Res. 50: $160-172$.

15. Ramamoorthi M, M Bakkar, J Jordan, and SD Tran. (2015). Osteogenic Potential of Dental Mesenchymal Stem Cells in Preclinical Studies: A Systematic Review Using Modified ARRIVE and CONSORT Guidelines. Stem Cells Int. 2015: 378368.

16. Shi S, PM Bartold, M Miura, BM Seo, PG Robey, and S Gronthos. (2005). The efficacy of mesenchymal stem cells to regenerate and repair dental structures. Orthod Craniofac Res. 8: 191-199.

17. Huang GT, S Gronthos, and S Shi. (2009). Mesenchymal stem cells derived from dental tissues vs. those from other sources: their biology and role in regenerative medicine. J Dent Res. 88: 792-806. 
18. Chadipiralla K, JM Yochim, B Bahuleyan, CY Huang, F Garcia-Godoy, PE Murray, and EJ Stelnicki. (2010). Osteogenic differentiation of stem cells derived from human periodontal ligaments and pulp of human exfoliated deciduous teeth. Cell Tissue Res. 340: 323-333. 19. Bakopoulou A, G Leyhausen, J Volk, A Tsiftsoglou, P Garefis, P Koidis, and W Geurtsen. (2011). Comparative analysis of in vitro osteo/odontogenic differentiation potential of human dental pulp stem cells (DPSCs) and stem cells from the apical papilla (SCAP). Arch Oral Biol. 56: 709-721.

20. Wang X, XJ Sha, GH Li, FS Yang, K Ji, LY Wen, SY Liu, L Chen, Y Ding, and K Xuan. (2012). Comparative characterization of stem cells from human exfoliated deciduous teeth and dental pulp stem cells. Arch Oral Biol. 57: 1231-1240.

21. Hakki SS, SA Kayis, EE Hakki, SB Bozkurt, G Duruksu, ZS Unal, G Turac, and E Karaoz. (2015). Comparison of mesenchymal stem cells isolated from pulp and periodontal ligament. J Periodontol. 86: 283-291.

22. Somerman MJ, SY Archer, GR Imm, and RA Foster. (1988). A comparative study of human periodontal ligament cells and gingival fibroblasts in vitro. Journal of dental research. 67: 66-70.

23. About I, MJ Bottero, P De Denato, J Camps, JC Franquin, and TA Mitsiadis. (2000). Human dentin production in vitro. Exp Cell Res. 258: 33-41.

24. Roura S, J Farre, C Soler-Botija, A Llach, L Hove-Madsen, JJ Cairo, F Godia, J Cinca, and A Bayes-Genis. (2006). Effect of aging on the pluripotential capacity of human CD105+ mesenchymal stem cells. Eur J Heart Fail. 8: 555-563.

25. Stolzing A, E Jones, D Mcgonagle, and A Scutt. (2008). Age-related changes in human bone marrow-derived mesenchymal stem cells: consequences for cell therapies. Mech Ageing Dev. 129: 163-173.

26. Javazon EH, KJ Beggs, and AW Flake. (2004). Mesenchymal stem cells: paradoxes of passaging. Exp Hematol. 32: 414-425.

27. Hanna H, LM Mir, and FM Andre. (2018). In vitro osteoblastic differentiation of mesenchymal stem cells generates cell layers with distinct properties. Stem cell research \& therapy. 9: 203-203.

28. Russell KC, DG Phinney, MR Lacey, BL Barrilleaux, KE Meyertholen, and KC O'connor. (2010). In vitro high-capacity assay to quantify the clonal heterogeneity in trilineage potential of mesenchymal stem cells reveals a complex hierarchy of lineage commitment. Stem Cells. 28: 788-798.

29. Waddington RJ, SJ Youde, CP Lee, and AJ Sloan. (2009). Isolation of distinct progenitor stem cell populations from dental pulp. Cells Tissues Organs. 189: 268-274.

30. Xu J, W Wang, Y Kapila, J Lotz, and S Kapila. (2009). Multiple differentiation capacity of STRO-1+/CD146+ PDL mesenchymal progenitor cells. Stem cells and development. 18: 487-496.

31. Mantesso A and P Sharpe. (2009). Dental stem cells for tooth regeneration and repair. Expert Opin Biol Ther. 9: 1143-1154.

32. Sharpe PT. (2016). Dental mesenchymal stem cells. Development. 143: 2273-2280.

33. Isaka J, A Ohazama, M Kobayashi, C Nagashima, T Takiguchi, H Kawasaki, T Tachikawa, and K Hasegawa. (2001). Participation of periodontal ligament cells with regeneration of alveolar bone. J Periodontol. 72: 314-323.

34. Carinci F, G Papaccio, G Laino, A Palmieri, G Brunelli, R D'aquino, A Graziano, V Lanza, L Scapoli, M Martinelli, and F Pezzetti. (2008). Comparison between genetic portraits of osteoblasts derived from primary cultures and osteoblasts obtained from human pulpar stem cells. J Craniofac Surg. 19: 616-625; discussion 626-617.

35. Tatullo M, G Falisi, M Amantea, C Rastelli, F Paduano, and M Marrelli. (2015). DENTAL PULP STEM CELLS AND HUMAN PERIAPICAL CYST MESENCHYMAL 
STEM CELLS IN BONE TISSUE REGENERATION: COMPARISON OF BASAL AND OSTEOGENIC DIFFERENTIATED GENE EXPRESSION OF A NEWLY DISCOVERED MESENCHYMAL STEM CELL LINEAGE. J Biol Regul Homeost Agents. 29: 713-718. 36. Nakamura S, Y Yamada, W Katagiri, T Sugito, K Ito, and M Ueda. (2009). Stem cell proliferation pathways comparison between human exfoliated deciduous teeth and dental pulp stem cells by gene expression profile from promising dental pulp. J Endod. 35: 15361542.

37. Lian JB and GS Stein. (1995). Development of the osteoblast phenotype: molecular mechanisms mediating osteoblast growth and differentiation. The Iowa orthopaedic journal. 15: 118-140.

38. Kaukua N, M Chen, P Guarnieri, M Dahl, ML Lim, T Yucel-Lindberg, E Sundstrom, I Adameyko, JJ Mao, and K Fried. (2015). Molecular differences between stromal cell populations from deciduous and permanent human teeth. Stem Cell Res Ther. 6: 59. 39. Khoshhal M, I Amiri, and L Gholami. (2017). Comparison of in vitro properties of periodontal ligament stem cells derived from permanent and deciduous teeth. J Dent Res Dent Clin Dent Prospects. 11: 140-148.

40. Silverio KG, TL Rodrigues, RD Coletta, L Benevides, JS Da Silva, MZ Casati, EA Sallum, and FH Nociti, Jr. (2010). Mesenchymal stem cell properties of periodontal ligament cells from deciduous and permanent teeth. J Periodontol. 81: 1207-1215. 


\section{Supplemental information S1}

Each of the three cell types were seeded in 12 well plates at a density of $4 \times 10^{4} / \mathrm{ml}$ and grown to $70 \%$ confluence. Cells were then induced with the osteogenic media (time zero), as previously outlined.

At days 1 and 7 RNA extraction and cDNA synthesis were carried out. Real-time qPCR investigating dentin sialophosphoprotein (DSPP) expression was subsequently carried out. Normalisation of results were achieved by using the house-keeping genes Beta- 2 microglobulin (B2M) and glucuronidase beta (GUSB). Primer sequences are listed in Table 1. The relative gene expression was calculated by the $2^{-\Delta \Delta \mathrm{CT}}$ method. Results are reported as fold change in gene expression relative to the day 1 PDLSC.

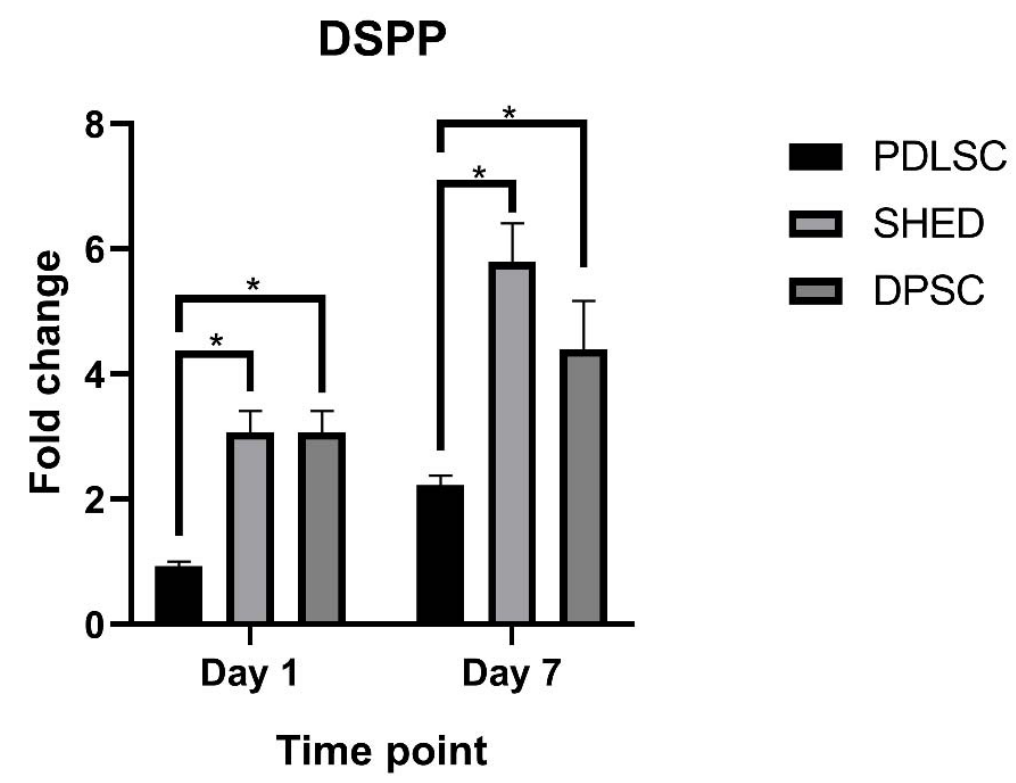

Supplemental Figure S1. Odontogenic-specific gene expression for DSPP in PDLSC, DPSC, and SHED. Odontogenic gene expression was assessed at Days 1 and 7. Levels of gene expression are provided as mean \pm SE fold change relative to the day 1 PDLSC gene expression levels, $\mathrm{n}=3 .{ }^{*} \mathrm{p}<0.05$ ( 2 way ANOVA - post-test Tukey’s). 
Figure and Figure legends

\section{Alkaline phosphatase activity}

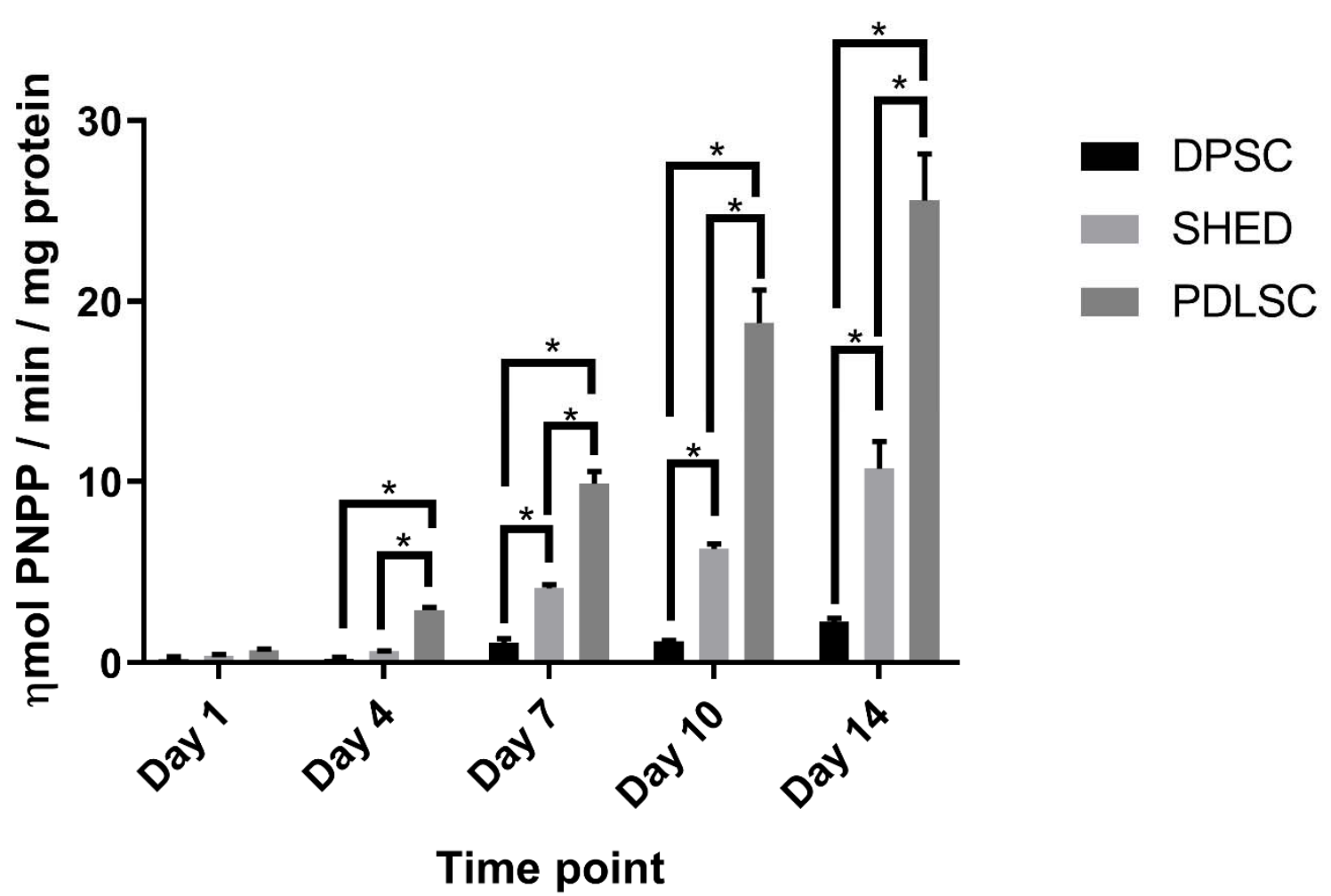

Figure 1. Alkaline phosphatase activity for DPSC, SHED and PDLSC at days 1, 4, 7, 10, and 14. Values were normalized to total protein content and expressed as mean \pm SE $\eta$ mol $\mathrm{PNPP} / \mathrm{min} / \mathrm{mg}$ protein, $\mathrm{n}=3 .{ }^{*} \mathrm{p}<0.05$ (Two-way ANOVA- post-test Tukey's) 


\begin{tabular}{|l|ll|ll|l|}
\hline & \multicolumn{2}{|c|}{ DAY 7 } & \multicolumn{2}{c|}{ DAY 14 } & \multicolumn{2}{c|}{ DAY 21 } \\
& Control Media & Osteogenic media & Control Media & Osteogenic media & Control Media Osteogenic media \\
\hline & & & & & \\
\hline \\
\end{tabular}

Figure 2. Alizarin red staining images for DPSCs, SHEDs, and PDLSCs cultured in both a control media and osteogenic media on days 7, 14, and 21. Representative well view. 


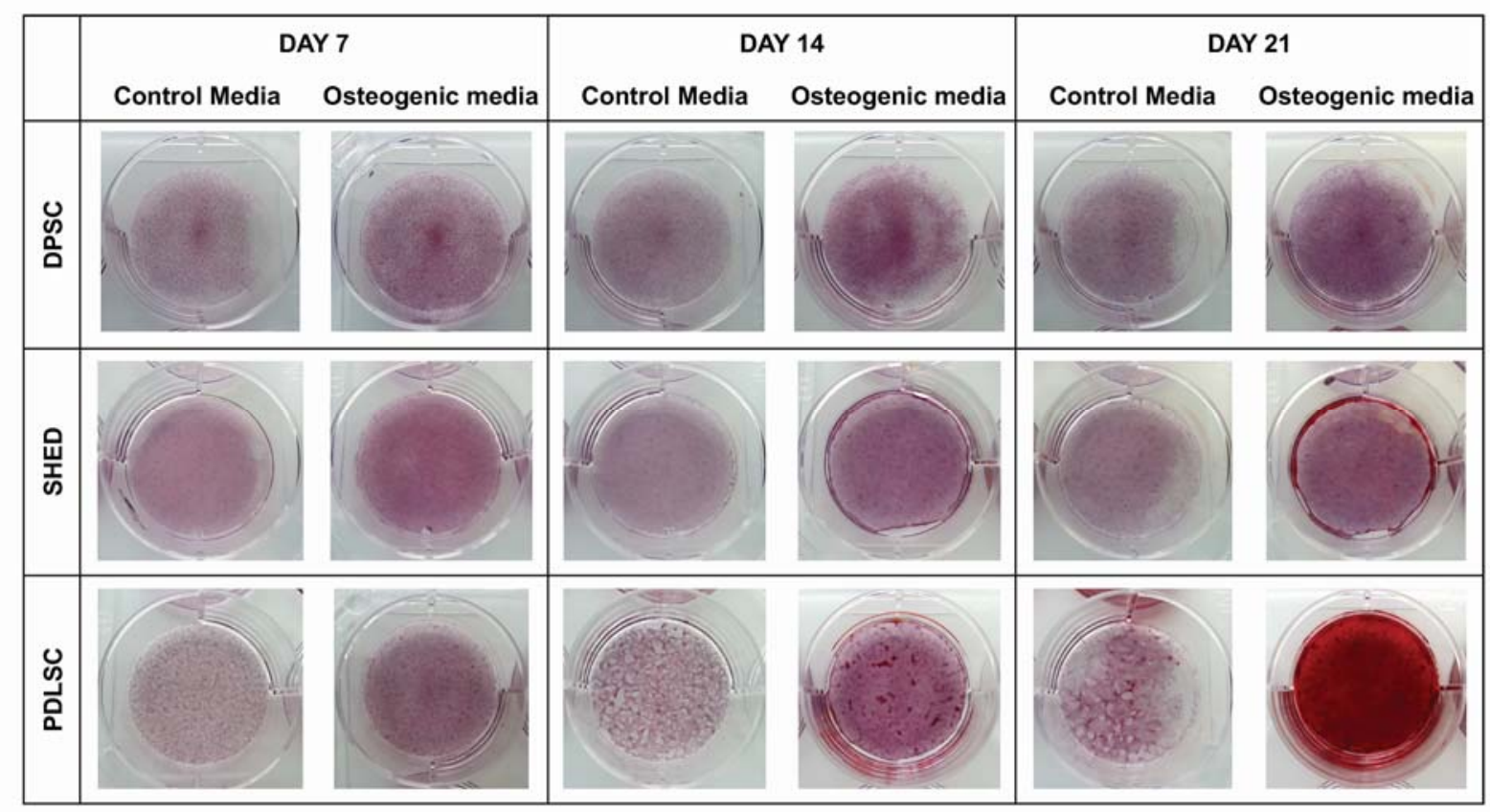

Figure 3. High magnification (x10) alizarin red staining images for DPSCs, SHEDs, and PDLSCs cultured in both a control media and osteogenic media on days 7, 14, and 21. 

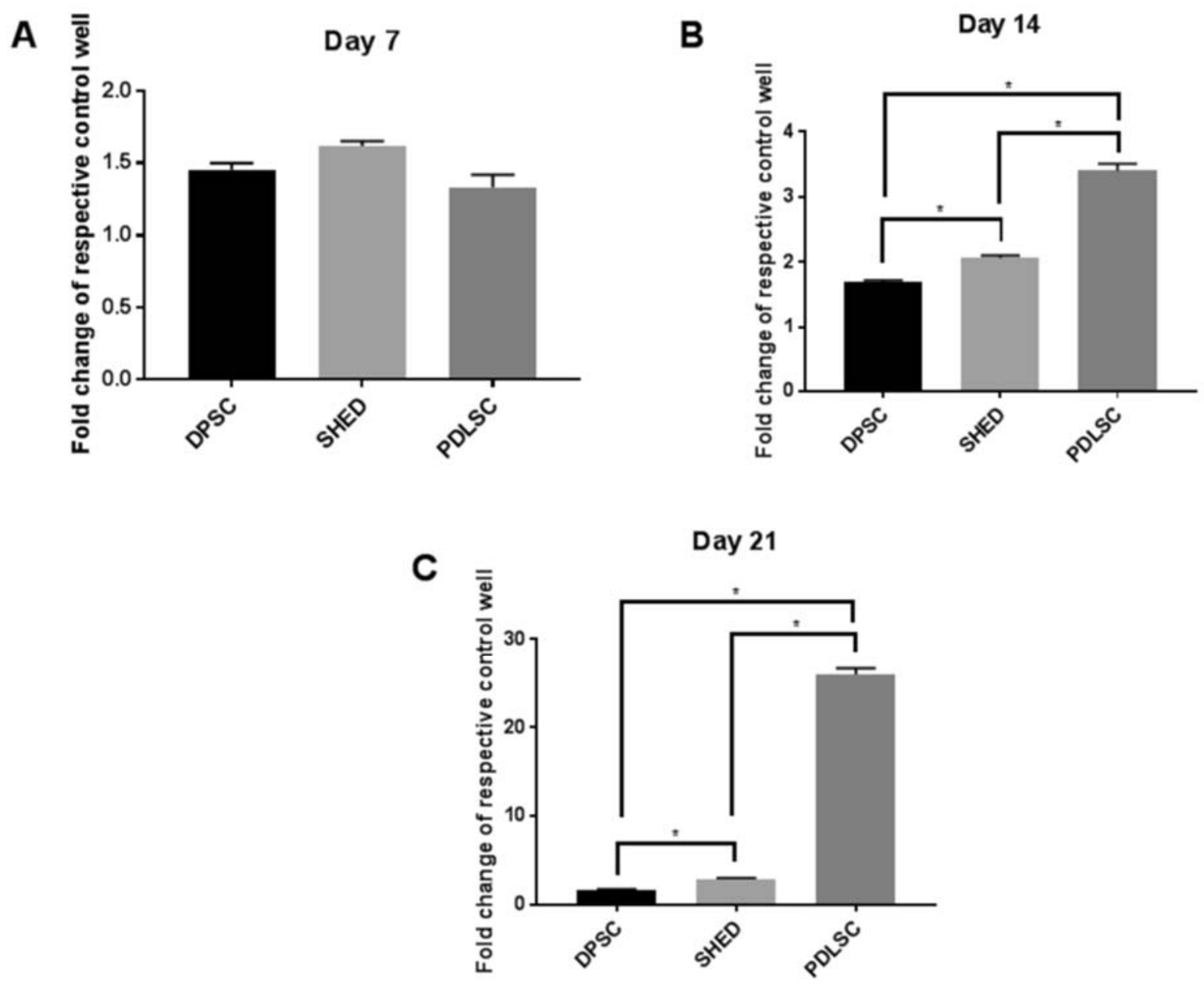

Figure 4. Alizarin Red staining quantification assay for DPSC, SHED and PDLSC at days 7 (A), 14 (B), and 21 (C). Values were reported as mean \pm SE fold change in optical density (at $570 \mathrm{~nm}$ ) in osteogenic media treated wells over their own respective control well, $\mathrm{n}=3 .{ }^{*} \mathrm{p}<0.05$ (One way ANOVA - post-test Tukey’s). 

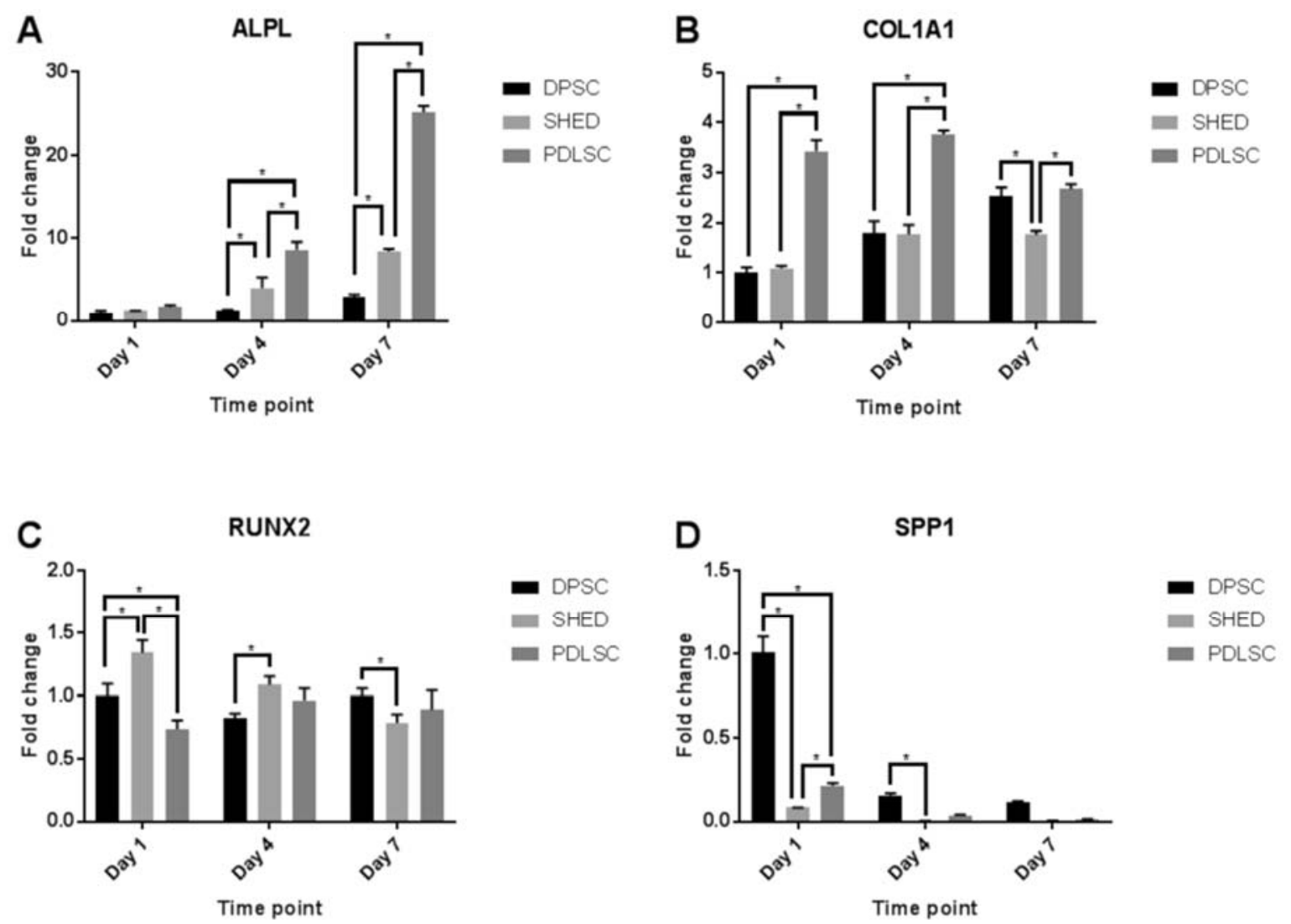

Figures 5. Bone-specific gene expression for ALPL (A), COL1A1 (B), RUNX2 (C), and SPP1 (D), in DPSC, SHED and PDLSC. Osteogenic gene expression was assessed at Days 1, 4, and 7. Levels of gene expression are provided as mean $\pm \mathrm{SE}$ fold change relative to the day 1 DPSC gene expression levels, $n=3 .{ }^{*} \mathrm{p}<0.05$ ( 2 way ANOVA - post-test Tukey's). 